\title{
Convergence analysis of the unpunctured turbo trellis-coded modulation (UTTCM)
}

\author{
Ahmed Haffane, Abdelhafid Hasni Mustapha Khelifi, Boufeldja Kadri \\ Laboratory of Information Processing and Telecommunications (LTIT), Tahri Mohammed University of Bechar, Algeria
}

\begin{tabular}{l}
\hline \hline Article Info \\
\hline Article history: \\
Received Sep 9, 2018 \\
Revised Nov 21, 2018 \\
Accepted Dec 15, 2018 \\
\hline
\end{tabular}

\section{Keywords:}

Channel coding

Convergence analysis

EXIT charts

UTTCM

\begin{abstract}
In this paper, the performance of the Unpunctured Turbo Trellis-Coded Modulation (UTTCM) over Additive White Gaussian Noise (AWGN) channel is analyzed using the non-binary extrinsic information transfer (EXIT) chart. The exchange of the extrinsic information between the decoder components is tracked, allowing the generation of an EXIT chart, which is a powerful tool for analyzing the convergence behavior of iterative decoding and prediction of convergence position. The Simulation results are compared with the turbo cliff positions on the BER curves.
\end{abstract}

Copyright (C) 2018 Institute of Advanced Engineering and Science. All rights reserved.

\section{Corresponding Author:}

Ahmed Haffane,

Laboratory of Information Processing and Telecommunications (LTIT),

Tahri Mohammed University of Bechar, Algeria.

Email: haffanhmed@gmail.com

\section{INTRODUCTION}

Turbo code as presented by Berrou and al [1] was the first error correcting code to closely approach the channel capacity. However, like other Error correcting codes they improve the reliability of data transmission at the expense of bandwidth expansion. The redundancy increases the number of bits within the transmitted symbol, which can lead to a reduction of information transmission rate or to an increase of the bandwidth. Trellis coded modulation (TCM) [2] is a bandwidth efficient coding techniques that integrates a convolutional code with a bandwidth-efficient modulation scheme.

Robertson and Wörz in 1995 [3] merge TCM and Turbo codes together in order to benefit from their both important structures properties and advantages, and proposed the "Turbo Trellis Coded Modulation" (TTCM). The encoder structure of the TTCM is made up of two identical TCM encoders in a parallel concatenation linked by a symbol interleaver. The coded symbols of each component encoder are alternatively punctured in order to increase the bandwidth efficiency. The decoding of TTCM is done iteratively using a symbol-by-symbol Maximum A Posteriori (MAP) component decoders. Derived from the TTCM but without punctuation, the Unpunctured Turbo Trellis Coded Modulation (UTTCM) [4], [5] is a more recent coded modulation technique, which transmits together: the input information bits and the 2 parity check bits from the two component coders in the same codeword.

Extrinsic Information Transfer (EXIT) chart [6] is powerful tool for analyzing the convergence properties of iterative decoding. EXIT chart was originally developed for concatenated binary coding schemes and expanded later to non-binary coding schemes [7], [8]. Based on the approach presented in [8], the purpose of this paper is to apply the EXIT chart to analyze the performance of the UTTCM. The rest of the paper is organized as follows: Section II describes the UTTCM, Section III presents the non-binary extrinsic information transfer characteristics. Simulation results and interpretations are presented in Section IV. The conclusion is reported in Section V. 


\section{UNPUNCTURED TRELLIS-CODED MODULATION}

The UTTCM turbo encoder as shown in Figure 1, as presented in [4], is a parallel concatenation of tow $m / m+1$ rate RSC (Recursive Systematic Convolution) encoders separated by an interleaver which can be an S-random interleaver or a pseudorandom interleaver [9]. Each RSC produces one parity bit for each $m$ systematic bits. The mapper takes the $m$ systematic bits, one parity bit from the upper encoder and the deinterleaved parity bit from the bottom encoder and maps them to $2^{m+2}$ constellation points.

Two mapping techniques were proposed. When the first mapping technique adopts Gray mapping, the second combines both natural mapping and Gray mapping, it is called Ungerboeck-Gray mapping [5] and it show good performances also when used in TCM, as shown in Figure 2 [10].

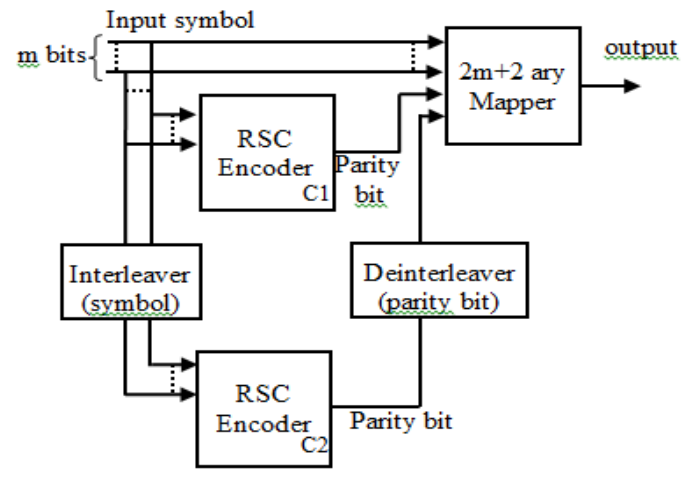

Figure 1. Structure of the unpunctured turbo trellis-coded modulation encoder [4]

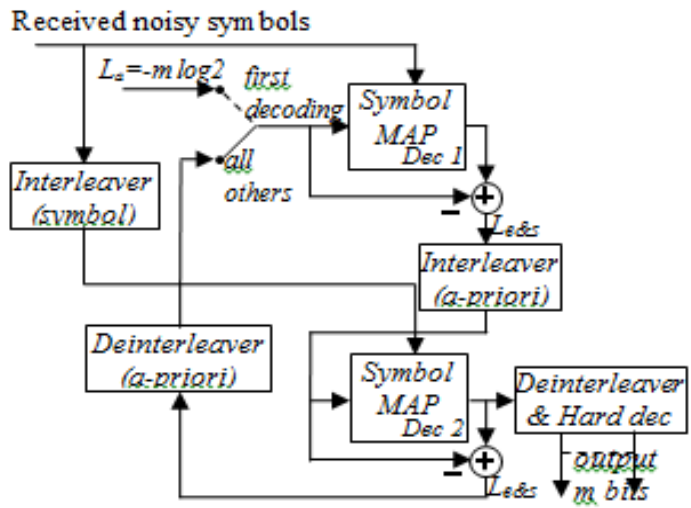

Figure 2. Decoding scheme of the unpunctured turbo trellis-coded modulation [4]

The structure of UTTCM receiver is similar to that of TTCM; both are serial concatenation of two constituent symbol-by-symbol MAP decoders [4] The objective of the decoder is to determine the A Posteriori Probabilities (APP) of the transmitted symbols over an iterative decoding process. The combined extrinsic and systematic information $\left(\mathrm{L}_{\mathrm{e} \& s}\right)$ is extracted from the output of one decoder and passed to the second decoder as a priori information $\left(\mathrm{L}_{\mathrm{a}}\right)$ in order to improve the reliability of the second decoder's output and vice versa. In this iterative decoding process, the apriori information is always available for the constituent decoders except for the first decoding stage were the only information available is that the probability of occurrence of one of the $2^{\mathrm{m}}$ combinations of the encoded symbol is equiprobable. Thus, the apriori information will be initialized with [5]:

$$
\operatorname{Pr}\left(d_{k}=i\right)=\left(\frac{1}{2}\right)^{m}
$$
received.

After a few iterations, a decision is conducted on the final APP to evaluate the symbol that was 
At each time $\mathrm{k}(\mathrm{k}=1,2, \ldots, \mathrm{L})$; the MAP decoder computes the A Posteriori Probability $\mathrm{A}_{\mathrm{k}, \mathrm{i}}$ that the information symbol $\mathrm{dk}$ was $\mathrm{i}\left(\mathrm{i}=0,1, \ldots, 2^{\mathrm{m}-1}\right)$ given the received sequence $\underline{y}$ and select the symbol with the largest APP. Thus, each decoder computes

$$
\operatorname{Pr}\left\{d_{k}=i \mid \underline{y}\right\}=\text { const } \sum_{s} \sum_{s^{\prime}} \gamma_{i}\left(y_{k}, s, s^{\prime}\right) \cdot \alpha_{k-1}\left(s^{\prime}\right) \cdot \beta_{k}(s)
$$

Where, the term (const) is a constant and it is canceled out by the normalization of (2) over all i to unity,

$\mathrm{S}_{\mathrm{k}}=\mathrm{s}$ is the present state of the encoder trellis and $\mathrm{S}_{\mathrm{k}-1}=\mathrm{s}^{\prime}$ is the previous state

$\alpha_{\mathrm{k}}\left(\mathrm{s}^{\prime}\right)$ is the forward metrics computed as

$$
\alpha_{k}(s)=\sum_{\text {all s' }} \gamma_{k}\left(s, s^{\prime}\right) \cdot \alpha_{k-1}\left(s^{\prime}\right)
$$

with the initial condition

$$
\begin{aligned}
& \alpha_{0}(0)=1 \\
& \alpha_{0}(s)=0 \text { for all } \mathrm{s} \neq 0
\end{aligned}
$$

$\beta_{\mathrm{k}}(\mathrm{s})$ is the backward metrics computed as

$$
\beta_{k-1}(s)=\sum_{\text {all } s^{\prime}} \gamma_{k}\left(s, s^{\prime}\right) \cdot \beta_{k}\left(s^{\prime}\right)
$$

with the initial condition

$$
\begin{aligned}
& \beta_{N}(0)=1 \\
& \beta_{N}(s)=0 \text { for all } \mathrm{M} \neq 0
\end{aligned}
$$

$\gamma_{\mathrm{k}}\left(\mathrm{y}_{\mathrm{k}}, \mathrm{s}, \mathrm{s}^{\prime}\right)$ is the branch transition metrics given by [5]

$$
\begin{aligned}
& \gamma_{k}\left(y_{k}, s, s^{\prime}\right)=p\left(y_{k} \mid d_{k}=i, S_{k}=s, S_{k-1}=s^{\prime}\right) . \\
& q\left(d_{k}=i \mid S_{k}=s, S_{k-1}=s^{\prime}\right) \cdot \operatorname{Pr}\left(S_{k}=s, S_{k-1}=s^{\prime}\right)
\end{aligned}
$$

The term $\mathrm{q}\left(\mathrm{d}_{\mathrm{k}}=\mathrm{i} \mid \mathrm{S}_{\mathrm{k}}=\mathrm{s}, \mathrm{S}_{\mathrm{k}-1}=\mathrm{s}^{\prime}\right)$ is equal to zero or one, depending on whether the input symbol $d_{k}=i$ induces a transition from state $s^{\prime}$ to $s$ or not and $\operatorname{Pr}\left(S_{k}=s, S_{k-1}=s^{\prime}\right)$ is set to the a priori probability of that symbol.

In the first term of (7), $\mathrm{y}_{\mathrm{k}}$ is the noisy version of the transmitted symbol constituted from $\mathrm{m}$ systematic bits, the parity bit generated by the one encoder and parity bit generated by the other encoder.

If $c_{\mathrm{k}}^{2} \in\{0,1\}$ is the other encoder parity bit generated at step $\mathrm{k}$, the probability $\mathrm{p}\left(\mathrm{y}_{\mathrm{k}} \mid \mathrm{d}_{\mathrm{k}}=\mathrm{i}, \mathrm{s}_{\mathrm{k}}=\mathrm{s}, \mathrm{s}_{\mathrm{k}-1}=\mathrm{s}^{\prime}\right)$ is set to

$$
\begin{aligned}
& p\left(y_{k} \mid d_{k}=i, s_{k}=s, s_{k-1}=s^{\prime}\right)=\sum_{j \in\{0,1\}} p\left(y_{k}, c_{k}^{2}=j \mid d_{k}=i, s_{k}=s, s_{k-1}=s^{\prime}\right)= \\
& \frac{1}{2} \sum_{j \in\{0,1\}} p\left(y_{k} \mid d_{k}=i, s_{k}=s, s_{k-1}=s^{\prime}, c_{k}^{2}=j\right)
\end{aligned}
$$

where it is assumed that

$$
p\left(c_{k}^{2}=j \mid d_{k}=i, s_{k}=s, s_{k-1}=s^{\prime}\right)=p\left(c_{k}^{2}=j\right)=\frac{1}{2}
$$

i.e.: the value of $c_{k}^{2}$ is statistically independent of $d_{k}, s_{k}$ and $s_{k-1}$ and can take either zero or one.

As in the case of TTCM the decoding algorithm can operate in the logarithmic domain and the LogMAP decoder is used or its improved algorithm [11], [12]

\section{CONVERGENCE PREDICTION USING EXIT CHART}

In an iterative decoding process, based on SISO decoders, the extrinsic information generated at the output of each decoder is passed to the input of the other one as apriori information. Over iterations, the extrinsic information at the input of the decoder enhances the knowledge about the transmitted symbols. To quantify the information that tells the extrinsic information about the transmitted symbols, the mutual information is used. Therefore for the decoding process to converge there must be an increase in mutual information across iterations. Thus, the mutual information "I $\mathrm{E}$ " computed for the extrinsic information at the 
output of each component decoder must be greater than the mutual information " $\mathrm{I}_{\mathrm{A}}$ " computed for the apriori information at its input.

Ten Brink [6] proposed to track the evolution of the extrinsic mutual information for an iterative decoding process on a single component decoder by substituting the apriori information -which should be produced at the output of the other decoder- by a vector generated based on a fixed apriori mutual information " $\mathrm{I}_{\mathrm{A}}$ " and to compute mutual information " $\mathrm{I}_{\mathrm{E}}$ " of the extrinsic information generated at its output. This operation is repeated for different values of " $\mathrm{I}_{\mathrm{A}}$ ". The resulting " $\mathrm{I}_{\mathrm{E}}$ " values are represented as a function of " $\mathrm{I}_{\mathrm{A}}$ " on a curve called the extrinsic information transfer characteristic. In order to flow the exchange of extrinsic information in an iterative decoding process, both decoder characteristics are plotted into a single diagram in which the axes of the transfer characteristics of the second decoder are swapped. The resulting diagram is the Extrinsic Information Transfer (EXIT) chart.

\subsection{Exit chart for UTTCM}

In the case of the UTTCM we follow the method describe in [13] to generate the vector of the apriori symbol probabilities.

Assuming that the binary bits constituting each transmitted symbol are mutually independent, the a priori symbol probabilities are generated via the observation of the information symbol over a multidimensional Gaussian channel. Namely, every bit from each symbol is BPSK modulated and then transmitted through an independent AWGN channel. So the a priori probability of each bit within each symbol is generated as for the binary case according to [6], then the obtained log-likelihood values are converted to symbol probability, according to:

$$
P\left(d=\left(b_{1}, b_{2}, \ldots, b_{m}\right)\right)=\prod_{j=1}^{m} \frac{e^{b_{j} L_{j}}}{1+e^{L_{j}}}
$$

Where $d$ is the encoded symbol formed from the $m$ information bits $b_{j} . L_{j}$ is the apriori $\log$ likelihood values of the bit $b_{j}$

The extrinsic probabilities must be evaluated at the output of the decoder. But, in the case of the UTTCM the systematic bits and the two parity bits are jointly transmitted through the channel, thus the extrinsic and the systematic information cannot be separated [3]. However, in [7] it is assumed that like the case of the binary turbo coding, the systematic information represents the information relative to the channel observation of the systematic bits. Therefore, it can be evaluated and subtracted along with the apriori information from the a posteriori symbol probability at the output of the decoder to compute the extrinsic part.

Yet, in our case the channel observation is associated also with the two parity bits.

So, supposing that the transmitted data symbols are equally likely, the systematic information about the symbol $\mathrm{d}_{\mathrm{k}}$ given by [7] $\left\{\mathrm{P}\left(\mathrm{d}_{\mathrm{k}}=\mathrm{i} \mid \mathrm{y}_{\mathrm{k}}\right)\right\}_{\mathrm{i}=0}^{2^{\mathrm{m}}-1}$. is computed as

$$
\left\{P\left(d_{k}=i \mid y_{k}\right)\right\}=\sum_{c_{k}^{1}=0}^{1} \sum_{c_{k}^{2}=0}^{1} P\left(y_{k} \mid s_{k}=\operatorname{map}\left(d_{k}=i \cdot c_{k}^{1} \cdot c_{k}^{2}\right)\right) .
$$

Where $s_{\mathrm{k}}$ is the codeword and $c_{\mathrm{k}}^{1}, c_{\mathrm{k}}^{2}$ are the two parity bits.

The corresponding average extrinsic mutual information will be calculated as [8]:

$$
I_{E}(d)=m+\frac{1}{L} \sum_{k=1}^{L} E\left[\sum_{i=0}^{2^{m}-1} e_{D}\left(d_{k}^{(i)}\right) \cdot \log _{2}\left(e_{D}\left(d_{k}^{(i)}\right)\right)\right]
$$

Where $\mathrm{e}_{\mathrm{D}}($.$) is the extrinsic probability and E[$.$] is the expectation, which can be approximated by$ simple time-averaging of the extrinsic probabilities of the information symbol.

Equation (12) is also used to calculate the average a priori information $I_{A}(d)$.

\section{RESULTS AND ANALYSIS}

In this section, the exit chart is used to investigate the performance of UTTCM over AWGN channel for 2 and 3 bps spectral efficiencies. The main advantage of the Exit chart is that the analysis is performed on a single decoder. No BER simulation of the iterative decoding scheme itself is required. However, this simulation is carried out for each case in order to verify the accuracy of the exit chart to predict the $\mathrm{E}_{\mathrm{b}} / \mathrm{N}_{0}$ convergence threshold. Figure 4 shows the resultant EXIT charts computed for 2 bps UTTCM system for various $\mathrm{E}_{\mathrm{b}} / \mathrm{N}_{0}$ values. The UTTCM encoder is made up of two identical 8-state rate $2 / 3$ recursive systematic convolution code using $(13,11,05)$ [5] as generator polynomials, a block length of 16384 symbols and 16QAM constellations with Gray mapping. At the receiver side, a symbol-by-symbol log-MAP decoding 
algorithm is used. From this figure it can be seen that for $\mathrm{E}_{\mathrm{b}} / \mathrm{N}_{0}=5.4 \mathrm{~dB}$, the two extrinsic information transfer characteristic of the two decoders intersect early. From this point of intersection, the mutual information at the output of both decoders will be poorer then the mutual information at their input, meaning that, after some iteration the decoder was no more able to produce extrinsic information with enhanced information on the transmitted bits. Therefore the iterative process will be unable to converge whatever is the number of iterations. For $\mathrm{E}_{\mathrm{b}} / \mathrm{N}_{0}=5.6 \mathrm{~dB}$ the two curves have just avoided the intersection before reaching the point $(1,1)$, meaning that, for this $\mathrm{E}_{\mathrm{b}} / \mathrm{N}_{0}$ and above the decoder produce an increased extrinsic information and thus the iterative process will converge to correct transmitted sequence with high probability. Hence, the convergence threshold is approximately $5.6 \mathrm{~dB}$. However, it can be clearly seen that the convergence threshold in BER curve of the Figure 3. is at $\mathrm{E}_{\mathrm{b}} / \mathrm{N}_{0}=6 \mathrm{~dB}$. Meaning that, there is a deference of $0.4 \mathrm{~dB}$ between the two results, which could be explain by the limit validity of the assumption that extrinsic information and the systematic information can be separated [8].

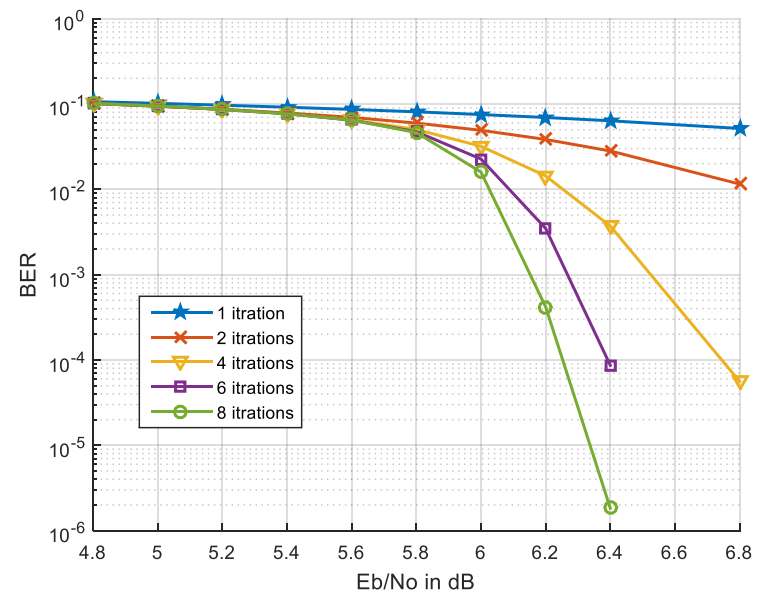

Figure 3. Bit error rate (BER) curves for UTTCM system (2 bps with 16-QAM a block length of 16384 symbols) at different decoding iterations

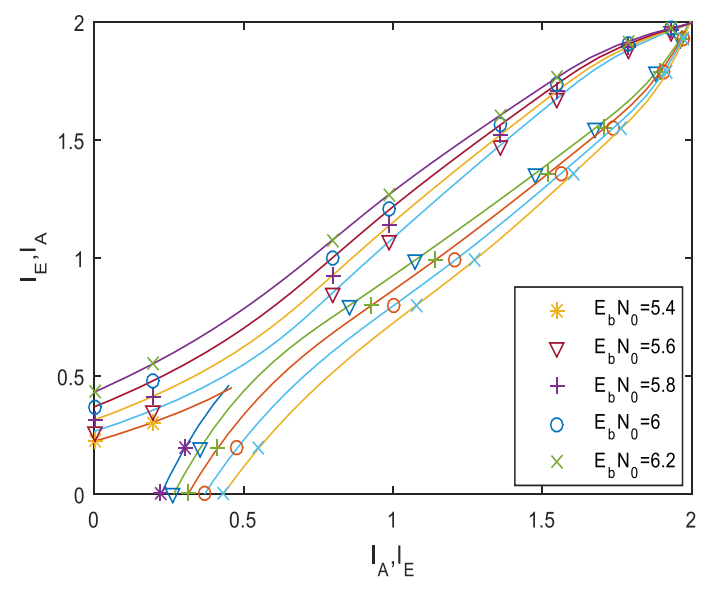

Figure 4. EXIT charts for UTTCM system (2 bps with 16-QAM and block length of 16384 symbols) at various $\mathrm{E}_{\mathrm{b}} / \mathrm{N}_{0}$ values

In Figure 6, the resultant EXIT charts computed for 3 bps UTTCM is depicted. The encoder is made up of two identical 8-state rate 3/4 recursive systematic convolution code using $(13,11,05,13)$ [5] as generator polynomials, 32-QAM constellations with Gray mapping, an interleaver length of 16384 symbols and a log-MAP decoder at the receiver side. The convergence is achieved for $E_{b} / N_{0}=10.4 \mathrm{~dB}$, with a $0.2 \mathrm{~dB}$ mismatch from the convergence threshold in BER curve of Figure 5, which may be again related to the assumption that extrinsic information and the systematic information can be separated [8].

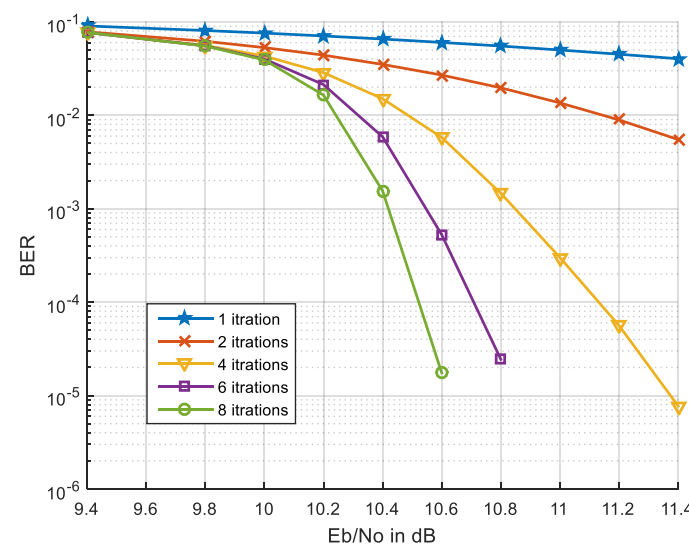

Figure 5. Bit error rate (BER) curves for UTTCM system (3 bps with 16-QAM a block length of 16384 symbols) at different decoding iterations

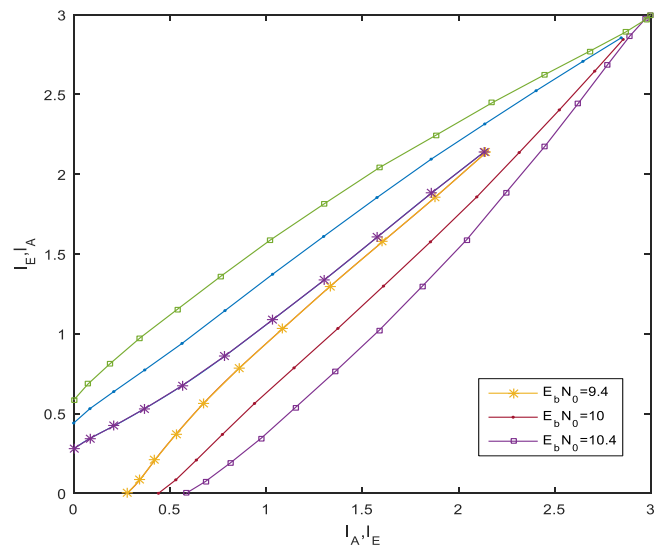

Figure 6. EXIT charts for UTTCM system (3 bps with 16-QAM and a block length of 16384 symbols) at various $\mathrm{E}_{\mathrm{b}} / \mathrm{N}_{0}$ values 


\section{CONCLUSION}

In this paper, the convergence behavior of iterative decoder of the Unpunctured Turbo Trellis Coded Modulation (UTTCM) using the non-binary EXIT chart was investigated. The accuracy of the exit chart to predict the decoding convergence threshold has been verified through examples. The results were compared to those of BER curve and have shown a slight mismatch, which is explained by the limited validity of the independence of the extrinsic information of each UTTCM emitted symbol from its systematic information. Nevertheless, the relatively less complicated method of generation makes the EXIT chart a more competitive technique for the comparison of different schemes as well as for the search of new designs.

\section{REFERENCES}

[1] Berrou C, Glavieux A and Thitimajshima P. Near Shannon limit error-correcting coding and decoding: Turbocodes. Proceedings of ICC '93 - IEEE International Conference on Communications, Geneva, Switzerland, 1993; 2: 1064-1070.

[2] Ungerboeck G. Channel coding with multilevel/phase signals. IEEE Transactions on Information Theory; 1982. 28(1): 55-67.

[3] Robertson P and Worz T. Bandwidth-efficient turbo trellis-coded modulation using punctured component codes. IEEE Journal on Selected Areas in Communications. 1998; 16(2): 206-218.

[4] Bassou A, Djebbari A. Efficient turbo encoding scheme using unpunctured trellis-coded modulation codes. $A E U$ International Journal of Electronics and Communications. 2007; 61(9): 621-626.

[5] Bassou A, Djebbari A, Benaissa M. Design of unpunctured turbo trellis-coded modulation. AEU-International Journal of Electronics and Communications. 2013; 67(3): 223-232.

[6] Brink S. ten. Convergence behavior of iteratively decoded parallel concatenated codes. IEEE Transactions on Communications. 2001; 49(10): 1727-1737.

[7] Chen H, Haimovich A. EXIT charts for turbo trellis-coded modulation. IEEE Communications Letters. 2004; 8(11): 668-670.

[8] Kliewer J, Ng S X, Hanzo L. Efficient Computation of EXIT Functions for Nonbinary Iterative Decoding. IEEE Transactions on Communications. 2006; 54(12): 2133-2136.

[9] Salim M, Yadav R P, Narwal K, Sharma A. A New Block S-Random Interleaver for Shorter Length Frames for Turbo Codes. Bulletin of Electrical Engineering and Informatics (BEEI), 2013; 2(4): 293-298.

[10] Rekkal K, Bassou A. Improving the Performance of Viterbi Decoder using Window System. International Journal of Electrical and Computer Engineering (IJECE), 2018,8(1): 611-621.

[11] Wang J, Li J, Cai C. Two novel decoding algorithms for turbo codes based on taylor series in 3GPP LTE system. Indonesian Journal of Electrical Engineering and Computer Science (IJEECS). 2014; 12(5):3467-3475.

[12] Wang J, Li J, Cai C. Novel Decoding Algorithm for BICM-ID Embedded Turbo Codes. Indonesian Journal of Electrical Engineering and Computer Science (IJEECS) , 2014; 12(5): 3460-3466.

[13] Grant A. Convergence of non-binary iterative decoding. GLOBECOM'01. IEEE Global Telecommunications Conference, San Antonio, TX. 2001; 2: 1058-1062. 